\title{
The Mechanism of Traditional Chinese Medicine for the Treatment of Obesity
}

This article was published in the following Dove Press journal:

Diabetes, Metabolic Syndrome and Obesity: Targets and Therapy

\section{Chang Li (D) \\ Hongli Zhang \\ Xiaohua Li (iD}

Department of Endocrinology, Seventh People's Hospital Affiliated to Shanghai University of TCM, Shanghai, People's Republic of China
Correspondence: Xiaohua Li

Department of Endocrinology, Seventh People's Hospital Affiliated to Shanghai University of TCM, Shanghai 200137,

People's Republic of China

$\mathrm{Tel} / \mathrm{Fax}+86$ 02I-5867056I

Email wendylee_tcm@shutcm.edu.cn

\begin{abstract}
Obesity is the lipid deposition caused by the imbalance between energy intake and consumption caused by a variety of factors. Obesity can lead to multiple systemic complications. At present, the treatment of obesity is mainly lifestyle intervention, drug weight loss, and weight loss surgery, but the curative effect is limited or the side effects are serious. Traditional Chinese medicine plays a unique role in the treatment of obesity. Existing studies have found that traditional Chinese medicine can treat obesity in a variety of ways, such as regulating intestinal microflora, enhancing hormone level, regulating fat metabolism, and so on. In this review, we will introduce and summarize the mechanism of traditional Chinese medicine in the treatment of obesity.
\end{abstract}

Keywords: metabolism, leptin, intestinal microflora, adiponectin

\section{Introduction}

According to the definition of the World Health Organization, overweight and obesity are defined as abnormal or excessive fat accumulation that may impair health. ${ }^{1}$ The prevalence of obesity among children, adolescents, and adults worldwide is increasing every year. ${ }^{2-4}$ According to the World Health Organization, the worldwide population rate of obesity (BMI $\geq 30$ ) in 2016 was significantly higher in all countries, especially the Americas, Europe, and Oceania. With the obesity rate in some countries as high as $60.7 \%$, the need for attention to this problem is urgent.

Obesity can lead to injury of multiple biological systems, significantly increasing the risk of metabolism-related diseases, cardiovascular diseases, respiratory diseases, Alzheimer's disease, osteoarthritis, and some cancers, ${ }^{5-8}$ and it increases mortality. ${ }^{9}$ To date, the main measures for treating obesity are lifestyle management, drugs for lipid reduction (orlistat and lipopeptide), weight loss surgery (Roux-en-Y gastric bypass, sleeve gastrectomy, or adjustable gastric banding) and strategies ${ }^{6}$ to reduce food intake and absorption and increase its utilization. However, due to a typical rebound in weight gain, the high risks associated with surgery and other problems, obesity has not been effectively resolved, and there is an urgent need to find a way to reduce the risks and improve patient compliance.

The ancient documentation of traditional Chinese medicine, "Lingshu Jing of the Inner Canon of Huangdi", pointed out that obesity means "people have fat, ointment and meat." It characterizes obesity as a disease mainly based on spleen and kidney deficiency and liver depression, with phlegm, dampness, heat, and blood stasis as the standard manifestations. Traditional Chinese medicine has been used 
for a long time for treating diseases, playing an important role in the treatment of obesity. ${ }^{10,11}$

In this paper, we selected drugs that have been widely discussed in obesity-related metabolic studies and can be used in clinical practice without obvious toxic and side effects, such as ginsenoside (Ginseng), ${ }^{12}$ celastrol (Tripterygium wilfordii), ${ }^{13}$ berberine (Coptis chinensis) ${ }^{14}$ curcumin (Turmeric), ${ }^{15}$ and the Xiexin ${ }^{16}$ and Danggui Buxue decoctions. ${ }^{17}$ Considering that different organ tissues may play similar weight-reducing roles under the role of TCM or TCM extracts in the weight-reducing process, we will mainly generalize from the specific mechanisms (regulating fat metabolism, intestinal Microflora, and regulating hormone level, etc.), as shown in Figure 1. In order to clarify the specific mechanism, the mechanism that plays the same role in different organs will be reflected in the tissue localization in Table 1. Summary of the mechanisms of traditional Chinese medicine and its extracts in the treatment of obesity, specifically as follows.

\section{Regulate Fat Metabolism}

It is well known that excessive energy intake or insufficient energy consumption by the human body can cause mature adipocytes to accumulate lipid and expand. The treatment of obesity can regulate the balance of energy intake and consumption by inhibiting lipid synthesis, promoting lipid decomposition, and/or promoting thermogenic fat activation. Traditional Chinese medicine has been successfully applied to the treatment of obesity in this way.

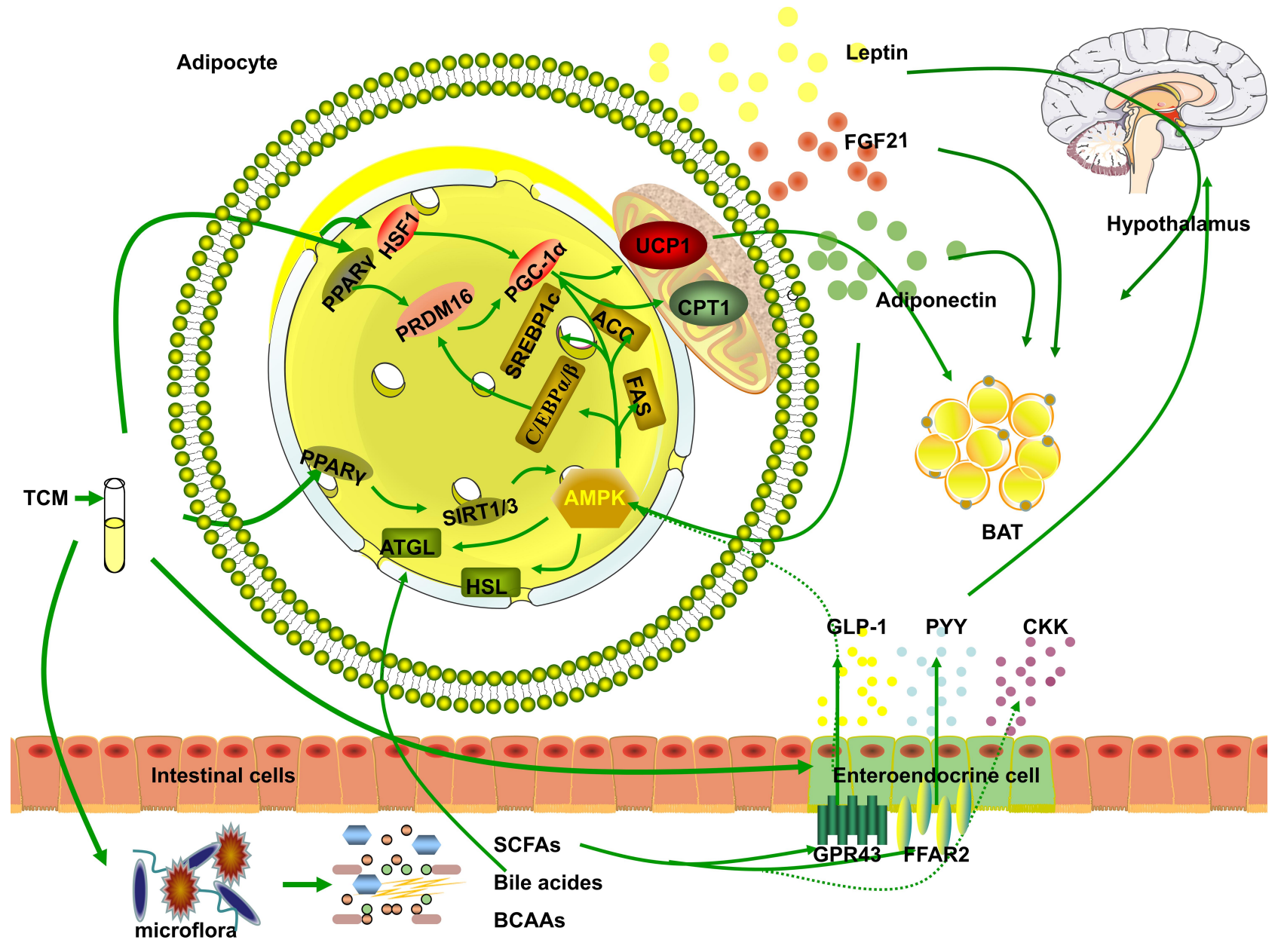

Figure I Basic Research on TCM in the Treatment of Obesity. The three pathways of TCM in obesity treatment to: I. By affecting the composition of intestinal microflora, regulation of intestinal metabolites (SCFAs, bile acids, and BACC) directly affects the roles of metabolites in obesity or indirectly increases the secretion of hormones by endocrine cells (GLP-I, PYY, and CKK) that regulate the hypothalamus, affect appetite and attenuate obesity. 2. AMPK is activated to regulate lipid metabolism or upregulate BAT-related genes in WAT to promote the browning of WAT and thermogenic fat activation. 3. Directly or indirectly promote fat tissue secretion of leptin, adiponectin, FGF2I, GLP-I, and PYY to promote lipid metabolism and/or affect appetite by regulating the hypothalamus. 
Table I Mechanism of TCM in the Treatment of Obesity

\begin{tabular}{|c|c|c|c|c|}
\hline TCM & Functions & Genes/Proteins & $\begin{array}{l}\text { Tissue } \\
\text { Localization }\end{array}$ & References \\
\hline \multirow{2}{*}{$\begin{array}{l}\text { Celastrol } \\
\text { (Tripterygium } \\
\text { wilfordii) }\end{array}$} & $\begin{array}{l}\text { Promote thermogenic fat } \\
\text { activation }\end{array}$ & HSFI, PGCI $\alpha$, UCPI $\uparrow$ & WAT; Muscle & $\left(\right.$ Ma et al, 2015) ${ }^{13}$ \\
\hline & Enhance leptin sensitivity & STAT3 $_{\text {Tyr705 }}$, SOCS3 $\uparrow$ & Hypothalamic & (Liu et al, 2015) (8 $^{68}$ \\
\hline \multirow[t]{3}{*}{$\begin{array}{l}\text { Capsaicin (Chili } \\
\text { pepper) }\end{array}$} & $\begin{array}{l}\text { Promote thermogenic fat } \\
\text { activation }\end{array}$ & $\begin{array}{l}\text { TRPVI, PPAR } \gamma-\text { PRDMI } 6 \rightarrow \\
\text { PGCI } \alpha-U C P I \uparrow\end{array}$ & EAT; SAT; Liver & $\begin{array}{l}\text { (Baskaran et al, 2016; } \\
\text { Bort et al, 2019) }\end{array}$ \\
\hline & Up-regulation of GLP-I andPYY & $\begin{array}{l}\text { PYY, GLP- } 1 \uparrow \rightarrow \\
\text { NPY- and AgRP-expressing neurons } \downarrow \text {, } \\
\text { POMC- and CART-expressing neurons } \uparrow \text {, }\end{array}$ & Hypothalamic & (Wang et al, 2020) $)^{46}$ \\
\hline & Increase the level of SCFAs & Acetate, propionate $\uparrow$ & Intestines & $\left(\right.$ Wang et al, 2020) ${ }^{46}$ \\
\hline \multirow[t]{3}{*}{$\begin{array}{l}\text { Chrysophanic Acid/ } \\
\text { Rhein (Rhubarb) }\end{array}$} & $\begin{array}{l}\text { Promote thermogenic fat } \\
\text { activation }\end{array}$ & $\begin{array}{l}\text { Sirtl, Sirt } 3 \uparrow \rightarrow \\
\text { AMPK } \uparrow, U C P I, P G C l \alpha \uparrow\end{array}$ & BAT & $(\text { Lim et al, 2016) })^{31}$ \\
\hline & $\begin{array}{l}\text { Inhibit fat production and } \\
\text { accumulation }\end{array}$ & $\mathrm{AMPK} \uparrow \rightarrow \operatorname{PPAR} \gamma, \mathrm{C} / \mathrm{EBP} \alpha$, GLUT4, Lipin I, AP $2 \downarrow$ & Preadipocytes & $(\text { Lim et al, 2016) })^{31}$ \\
\hline & Up-regulation of GLP-I & GLP-I $\uparrow$ & $\begin{array}{l}\text { Small intestine; } \\
\text { lleum }\end{array}$ & (Wang et al, 2018) ${ }^{86}$ \\
\hline \multirow{2}{*}{$\begin{array}{l}\text { Resveratrol } \\
\text { (Polygonum } \\
\text { cuspidatum) }\end{array}$} & $\begin{array}{l}\text { Inhibit fat production and } \\
\text { accumulation }\end{array}$ & SREBP-IC $\downarrow$ & Liver & $\left(\right.$ Izdebska et al, 2018) ${ }^{28}$ \\
\hline & Regulation of bile acid excretion & CYP7AI $\uparrow$ & $\begin{array}{l}\text { Liver; } \\
\text { Duodenum; } \\
\text { lleum }\end{array}$ & (Chambers et al, 2019) $^{61}$ \\
\hline \multirow{2}{*}{$\begin{array}{l}\text { INDUS8I0 } \\
\text { (Trigonella foenum- } \\
\text { graecum (L.)) }\end{array}$} & $\begin{array}{l}\text { Inhibit fat production and } \\
\text { accumulation }\end{array}$ & $\begin{array}{l}\text { AMPK } \uparrow \\
\operatorname{PPAR} \beta, \operatorname{PGC} \beta, \text { SIRTI, SIRT } 3 \uparrow\end{array}$ & $\begin{array}{l}\text { WAT(3T3); } \\
\text { Liver }\end{array}$ & $\left(\right.$ Cheng et al, 2018) ${ }^{19}$ \\
\hline & Increase lipid decomposition & AMPK, ATGL $\uparrow$ & $\begin{array}{l}\text { Adipose tissue; } \\
\text { Liver }\end{array}$ & $(\text { Cheng et al, 2018) })^{19}$ \\
\hline \multirow{2}{*}{$\begin{array}{l}\text { Tea catechins } \\
\text { GT-polyphenols } \\
\text { (Green tea) }\end{array}$} & Increase lipid decomposition & $\begin{array}{l}\text { ACO } \uparrow \text { : MCAD } \uparrow \rightarrow \text { oxidation of FAs, } \\
\text { AMPKal } \uparrow \rightarrow \text { PKA-HSL } \uparrow\end{array}$ & Liver; SAT & $\begin{array}{l}\text { (Murase et al,2002; Bolin } \\
\text { et al,2020) }\end{array}$ \\
\hline & Regulate the level of adiponectin & adiponectin $\uparrow$ & WAT; BAT & $\left(\right.$ Bolin et al,2020) ${ }^{79}$ \\
\hline \multirow[t]{2}{*}{ Xiexin decoction } & $\begin{array}{l}\text { Inhibit fat production and } \\
\text { accumulation }\end{array}$ & $\begin{array}{l}\text { AMPK } \uparrow, \\
\text { SREBPs, ACC, FAS } \downarrow\end{array}$ & Liver & $(\text { Xiao et al, 2019) })^{16}$ \\
\hline & Increase the level of SCFAs & $\begin{array}{l}\mathrm{ACK}, \mathrm{MMD}, \mathrm{BUT}, \mathrm{BUK} \uparrow \\
\rightarrow \mathrm{SCFAs} \uparrow\end{array}$ & Colon & $(\text { Xiao et al, 2019) })^{16}$ \\
\hline \multirow[t]{2}{*}{$\begin{array}{l}\text { White tiger plus } \\
\text { ginseng decoction }\end{array}$} & $\begin{array}{l}\text { Inhibit fat production and } \\
\text { accumulation }\end{array}$ & AMPK $\uparrow, A C C \downarrow$ & Liver & $($ Liu et al, 2015) \\
\hline & Increase lipid decomposition & AMPK, ATGL $\uparrow$ & Liver & $(\text { Liu et al, 2015) })^{21}$ \\
\hline \multirow[t]{4}{*}{$\begin{array}{l}\text { Curcumin } \\
\text { (Turmeric) }\end{array}$} & $\begin{array}{l}\text { Promote thermogenic fat } \\
\text { activation }\end{array}$ & $\begin{array}{l}\text { FGF2 I, Cidea, Tmem26, Tbxl, UCPI, PGC-I } \alpha, \text { PRDMI6, } \\
\text { C/EBP } \beta, \text { CPTI, Oxidation of fatty acids } \uparrow\end{array}$ & SAT & $(\text { Lone et al, 2016) })^{30}$ \\
\hline & $\begin{array}{l}\text { Inhibit fat production and } \\
\text { accumulation }\end{array}$ & $\begin{array}{l}\mathrm{P}-\mathrm{ACC} \uparrow \\
\mathrm{ACC} \downarrow\end{array}$ & SAT & $(\text { Lone et al, 2016) })^{30}$ \\
\hline & Enhance leptin sensitivity & leptin sensitivity $\uparrow$ & EAT & (Chang et al, 2018) ${ }^{72}$ \\
\hline & Regulate the level of adiponectin & adiponectin $\uparrow$ & EAT & (Chang et al, 2018) ${ }^{72}$ \\
\hline
\end{tabular}

(Continued) 
Table I (Continued).

\begin{tabular}{|c|c|c|c|c|}
\hline TCM & Functions & Genes/Proteins & $\begin{array}{l}\text { Tissue } \\
\text { Localization }\end{array}$ & References \\
\hline \multirow{4}{*}{$\begin{array}{l}\text { Berberine (Coptis } \\
\text { Chinensis) }\end{array}$} & Increase the level of SCFAs & Acetate, propionate $\uparrow$ & Colon & (Zhang et al, 20I2) ) $^{45}$ \\
\hline & Increase lipid decomposition & AMPK, ATGL, P-HSL $\uparrow$ & WAT(3T3) & $(\text { Jiang et al, 2016) })^{20}$ \\
\hline & Regulation of $\mathrm{BCAA}$ & $\begin{array}{l}\mathrm{BCKDC} \uparrow, \mathrm{BCKDHA}, \mathrm{BCKDK} \downarrow \rightarrow \\
\mathrm{BCAA} \text {, valine, leucine, isoleucine } \downarrow\end{array}$ & Liver; EAT & (Yue et al, 2019) ${ }^{63}$ \\
\hline & $\begin{array}{l}\text { Increase the level of SCFAs and } \\
\text { GLP-I }\end{array}$ & SCFAs30, GPR43-GLP-I $\uparrow$ & $\begin{array}{l}\text { Colon; } \\
\text { Gastrointestinal }\end{array}$ & $\left(\right.$ Sun et al, 2018) ${ }^{14}$ \\
\hline \multirow[t]{2}{*}{$\begin{array}{l}\text { Chaihu Shugan } \\
\text { Powder }\end{array}$} & Enhance leptin sensitivity & leptin sensitivity $\uparrow$ & $\begin{array}{l}\text { Adipose tissue; } \\
\text { Liver }\end{array}$ & $\left(\right.$ liang et al, 2018) ${ }^{73}$ \\
\hline & Regulate the level of adiponectin & adiponectin $\uparrow$ & $\begin{array}{l}\text { Adipose tissue; } \\
\text { Liver }\end{array}$ & (Jiang et al, 2018) ${ }^{73}$ \\
\hline
\end{tabular}

Abbreviations: ACC, acetyl-CoA carboxylase; ACK, acetate kinase; ACO, acyl-COA oxidase; AgRP, agouti-related protein; AMPK, AMP-activated protein kinase; AP2, fatty acid-binding protein; ASIV, Astragaloside IV; ATGL, adipose triglyceride lipase; BCAAs, branched chain amino acids; BAS, bile acids; BAT, brown adipose tissues; BCKDC, branched-chain ketoacid dehydrogenase complex; BCKDHA, branched chain keto acid dehydrogenase EI $\alpha$ subunit; BCKDK, branched-chain $\beta$-keto dehydrogenase kinase; BDNF, brain-derived neurotrophic factor; BeAT, beige adipose tissues; BUK, butyrate kinase; BUT, butyryl-CoA:acetate CoA transferase; C/EBP $\alpha / \beta$, CCAAT/ enhancer-binding protein $\alpha / \beta$; Cidea, cell death-inducing DNA fragmentation factor alpha-like effector A; CCK, cholecystokinin; Cox8b, cytochrome $\mathrm{c}$ oxidase 8b subunit; GPCR, G protein-coupled receptor; CPTI, carnitine palmitoyltransferase I; CYP7AI, cytochrome P450 family 7 subfamily A member I; DGATI/2, diacylglycerol O-acyltransferase I/2; DiO2, type II iodothyronine deiodinase; FAT, fatty acid translocase; FFAs, free-fatty-acids; FFAR2, free fatty acid receptor 2; FGF2I, fibroblast growth factor 2I; FXR, liver farnesol X receptor; GLP-I, glucagon-like peptide I; GLUT4, glucose transporter type 4; GPR43, G protein-coupled receptor 43; HFD, high-fat diet; HSFI, heat shock transcriptional factor I; HSL, hormone-sensitive lipase; IWAT, inguinal adipose tissue; JAK2, Janus kinase 2; LEPR, leptin receptor; L-SGgly, low-polar 22 Siraitia grosvenorii glycoside; $L X R \alpha$, liver $X$ receptor alpha; MCAD, medium-chain acyl-CoA dehydrogenase; MMD, methylmalonyl-CoA decarboxylase; mTOR, liver mechanistic target of rapamycin kinase; NPY, neuropeptide Y; NST, nonshivering thermogenesis; PGCl $\alpha / \beta$, peroxisome proliferator-activated receptor-gamma coactivator I alpha/beta; PPAR $\beta / \gamma$, peroxisome proliferator-activated receptor beta/gamma; PRDMI6, PR domain containing I6; PYY, peptide YY; SCD-I, stearoyl-CoA desaturase-I; SCFASs, short-chain fatty acids; SIRTI/3, sirtuin I/3; SNS, sympathetic nervous system; SREBPIc, sterol response element-binding protein Ic; STAT3, signal transducer and activator of transcription 3; TAG, triglyceride; TbxI, T-box transcription factor; TCM, traditional Chinese medicine; TGR5, G protein-coupled bile acid receptor I; Tmem26, transmembrane protein 26; TRPVI, transient receptor potential cation channel subfamily $V$ member I; UCPI, mitochondrial uncoupling protein I; WAT, white adipose tissues.

\section{Increase Lipid Decomposition}

Traditional Chinese medicine can increase the decomposition of lipids to treat obesity through the regulation of AMP-activated protein kinase (AMPK), autonomic nerves, or $\beta$-adrenoceptors. After being activated, AMPK can coordinate a variety of metabolic pathways (including liver lipid metabolism), regulate metabolism by phosphorylating key metabolic proteins and transcription factors, promote catabolism and inhibit anabolism. ${ }^{18}$ Many traditional Chinese medicines affect lipid synthesis and metabolism by acting on AMPK to induce weight loss. INDUS810 isolated from Trigonella foenum-graecum (L.) can activate AMPK to increase lipid degradation in a time-dependent manner. ${ }^{19}$ Berberine can upregulate the expression of adipose triglyceride lipase (ATGL) and phosphorylation of hormone-sensitive lipase (p-HSL) through AMPK, thus stimulating basal lipolysis in mature adipocytes. ${ }^{20}$ The phosphorylation of AMPK induced by the white tiger plus ginseng decoction promotes the expression of ATGL and lipolysis. ${ }^{21}$ The smell of grapefruit oil and its active ingredient limonene affects the autonomic nervous system and increases fat decomposition through a histamine reaction. ${ }^{22}$ The extract of Nelumbo nucifera leaves can upregulate the lipolysis of 3T3-L1 adipocytes as mediated by $\beta$-adrenoceptors. ${ }^{23}$ Long-term use of tea catechins (Green tea) could effectively activate the mRNA expression of acyl-COA oxidase (ACO) and medium-chain acyl-COA dehydrogenase (MCAD) in the liver, promote the oxidation of fatty acids, and inhibit the diet-induced obesity. ${ }^{24}$

\section{Inhibit Fat Production and Accumulation}

Obesity can lead to adipocyte proliferation and hypertrophy and thus can also play a role in weight loss by inhibiting the proliferation and differentiation of adipocytes. Hydroxy safflower yellow pigment (HSYA), the extract of Carthamus tinctorius, can inhibit the proliferation of 3T3-L1 preadipocytes in a time- and concentration-dependent manner. ${ }^{25}$ The ethanol extract of Aster yomena (EEAY) exerts its antiadipogenic effect by inhibiting or delaying the differentiation of 3T3-L1 preadipocytes and the expression of adipogenic factors (peroxisome proliferator-activated receptor gamma 
(PPAR $\gamma)$, CCAAT/enhancer-binding protein $\alpha / \beta(\mathrm{C} / \mathrm{EBP} \alpha / \beta)$ and sterol response element-binding protein 1c (SREBP1c)), inhibiting the de novo synthesis of lipids and the differentiation of adipocytes. ${ }^{26}$ The water extract from Ganoderma lucidum mycelia can reduce the expression of adipogenic genes and the level of serum free-fatty-acids (FFAs) in a dose-dependent manner in mice fed a high-fat diet (HFD). ${ }^{27}$ Resveratrol (Polygonum cuspidatum) and the extract from fermented Cordyceps militaris decrease the expression and activity of SREBP-1c and decrease fat synthesis. $^{28,29}$ Curcumin increases the phosphorylation of acetyl-CoA carboxylase (p-ACC) and decreases the ratelimiting enzyme acetyl-CoA carboxylase (ACC) of fatty acid synthesis, which inhibits lipogenesis. ${ }^{30}$ Traditional Chinese medicine can also inhibit lipid synthesis and metabolism by activating AMPK. Chrysophanic acid (rhubarb) can reduce weight by activating AMPK to inhibit lipid accumulation and the expression of adipogenic factors (PPAR $\gamma$ and $\mathrm{C} / \mathrm{EBP} \alpha$, glucose transporter type 4 (GLUT4), lipin 1 and adipocyte fatty acid-binding protein (AP2)) in 3T3-L1 adipocytes. $^{31}$ INDUS810 can reduce the content of intracellular triglycerides after adipocyte differentiation, reduce lipid accumulation during lipid differentiation in an AMPKdependent manner, and increase the expression of peroxisome proliferator-activated receptor beta (PPAR $\beta$ ), peroxisome proliferator-activated receptor-gamma coactivator 1 beta (PGC1 $\beta$ ), sirtuin 1 (SIRT1) and SIRT3. ${ }^{19}$ Babaodan can downregulate the expression of lipid synthesis genes (SREBP-1c, ACC, stearoyl-CoA desaturase-1 (SCD1), liver $X$ receptor alpha $(L X R \alpha)$, and $F A T / C D 36)$ by activating AMPK. ${ }^{32}$ The Xiexin decoction can also upregulate AMPK, inhibit SREBPs, downregulate the expression of ACC and fatty acid synthase (FAS), and reduce fat production. ${ }^{16}$ The white tiger plus ginseng decoction can induce the activation of AMPK, inhibit the expression of ACC, and reduce the synthesis and accumulation of lipids. ${ }^{21}$

\section{Promote Thermogenic Fat Activation}

Traditional Chinese medicine can promote the browning of white adipose tissue (WAT) by upregulating the expression of brown adipose tissue (BAT)-related genes in white adipose tissue or activating BAT heat generation to increase energy consumption and weight loss. Celastrol can induce the browning of inguinal adipose tissue (IWAT), upregulate heat shock transcriptional factor 1 (HSF1) expression, increase peroxisome proliferatoractivated receptor-gamma coactivator 1 alpha/mitochondrial uncoupling protein 1 (PGC1 $1 / \mathrm{UCP} 1)$ expression, and increase thermogenic function and energy consumption. ${ }^{13}$ Capsaicin (chili pepper) can induce the browning of WAT by activating transient receptor potential cation channel subfamily $\mathrm{V}$ member 1 (TRPV1) channels. $^{33,34}$ Chrysophanic acid increases the expression of the thermogenic genes $U C P 1$ and $P G C 1 \alpha$ by upregulating SIRT1 and SIRT3, activating AMPK and promoting thermogenesis. $^{31}$ Jiang Gui Fang (JG) increases the expression of BAT markers (PPAR $\gamma, \operatorname{PGC} 1 \alpha$, and UCP1), activates interscapular brown adipose tissue through the PPAR $\gamma /$ SIRT1-PGC1 $\alpha$ pathway, and induces IWAT browning. ${ }^{35}$ The Danggui Buxue decoction (DBT1155) can activate the Ca2+-AMPK signaling pathway in a time-dependent manner to trigger WAT browning in adipocytes and increase the expression of BAT markers in a dose-dependent manner. ${ }^{17}$ Curcumin can significantly upregulate the expression of BAT-specific genes (fibroblast growth factor 21 (FGF21), cell death-inducing DNA fragmentation factor alpha-like effector A (Cidea), transmembrane protein 26 (Tmem26), T-box transcription factor (Tbxl)) and proteins (UCP1, PGC1 $\alpha$ and PR domain containing 16 (PRDM16)) to drive BAT thermogenesis. Curcumin can also increase carnitine palmitoyltransferase I (CPT1) to increase the activity of mitochondria in brown adipocytes and enhance the oxidation of fatty acids. ${ }^{30}$ The water extract from Caulis Spatholobi (WECS) can promote whole-body energy metabolism by increasing the oxygen consumption of HFD mice and increasing the levels of BAT febrile genes (UCP1, PRDM16, and $P G C 1 \alpha$ and medium-chain acyl-CoA dehydrogenase $(M C A D))$ mRNA). ${ }^{36}$ Withania somnifera extract (WSE) increases oxygen consumption and enhances the expression of BAT genes, including UCP1, PGC1 , type II iodothyronine deiodinase (DiO2) and cytochrome c oxidase $8 b$ subunit (Cox $8 b$ ), and promotes the browning of subcutaneous adipose tissue in mice fed a high-fat $\operatorname{diet}^{37}$

\section{Regulate Intestinal Microflora}

Intestinal microflora can affect host energy metabolism, the immune system and the inflammatory response, and changes to its structure and function can promote obesity. By changing the abundance and constituent ratio of intestinal microorganisms, traditional Chinese medicine can increase the proportion and content of short-chain fatty acids (SCFAs), regulate the levels of bile acids, choline, amino acids, and other metabolites, thus playing a role in improving obesity. 


\section{Increase the Level of SCFAs}

SCFAs produced by intestinal microorganisms can activate G protein-coupled receptor 43 (GPR43) to inhibit insulin signal transduction in adipocytes, thereby inhibiting fat accumulation in adipose tissue and promoting unbound lipid metabolism in other tissues. ${ }^{38}$ Acetate, propionate, and butyrate account for $83 \%$ of human intestinal SCFAs. ${ }^{39}$ Acetate can activate AMPK to increase the indexes related to lipid catabolism, reduce abdominal lipids, and protect the liver and abdomen from lipid accumulation. ${ }^{40}$ Butyrate can reduce the accumulation of fat in the liver, reduce metabolism and mitochondrial efficiency, and counteract obesity. ${ }^{41}$ Propionic acid, similar to butyric acid, induces weight loss by adjusting the energy balance. ${ }^{42-44}$

Berberine and capsaicin can improve obesity by increasing acetic acid and propionic acid levels. ${ }^{45,46}$ Chinese cannabis can increase acetate levels to reduce visceral fat and induce weight loss. ${ }^{47}$ Protopanaxatriol saponins (PPTs) can increase the levels of SCFAs and their receptors to ameliorate obesity. ${ }^{48}$ Inulin can increase SCFAs in the colon and thus affect appetite and the secretion of peptide YY (PYY) and glucagon-like peptide 1 (GLP-1) to regulate obesityrelated metabolism. ${ }^{49}$ Homogenized polysaccharide S-3-1 from the Sijunzi decoction can improve the production of intestinal microbiota-derived SCFAs, regulate energy metabolism, and attenuate dyslipidemia induced by a high-fat diet in obese rats. ${ }^{50}$ The Xiexin decoction reduces weight by increasing the level and activity of key enzyme mRNAs involved in SCFA synthesis (acetate kinase (ACK), methylmalonyl-CoA decarboxylase (MMD), butyryl-CoA: acetate CoA transferase (BUT) and butyrate kinase (BUK)) and increasing the abundance of intestinal microflora. ${ }^{16}$

\section{Regulate the Levels of Bile Acids and Other Metabolites}

There is a two-way interaction between bile acid and intestinal microbial composition. ${ }^{51}$ Bile acid can change the bacterial community structure, affect host metabolism, ${ }^{52}$ regulate liver lipid, glucose and energy balance, and maintain metabolic balance. ${ }^{53}$ Bile acid can also induce WAT browning by activating $\mathrm{G}$ protein-coupled bile acid receptor 1 (TGR5) ${ }^{54}$ or directly or indirectly affect glucose metabolism and insulin signal transduction through lipid emulsification, affecting the cell membrane and inducing ectopic functions and receptors (such as liver farnesol $\mathrm{X}$ receptor (FXR) and G protein-coupled receptor (GPCR)). ${ }^{55}$ Choline can stimulate the lipolysis of triglyceride (TAG) in adipocytes and liver through specific lipases (ATGL, lipoprotein lipase (LPL) and HSL), inhibit the formation of TAG through diacylglycerol O-acyltransferase $1 / 2$ (DGAT1/2), activate liver AMPK and mitochondrial fatty acid oxidation gene PPAR $\alpha$, and reduce fatty acid synthesis genes SREBP1, SCD1 and FAS to reduce hepatic steatosis. ${ }^{56,57}$ It was found that inducing a reduction in the number of branched-chain amino acids (BCAAs) leads to rapid body weight and fat weight loss. ${ }^{58}$ BCAAs can inhibit lipid production and autophagy by activating the liver mechanistic target of rapamycin kinase (mTOR) and reduce HFD-induced body weight. ${ }^{59}$

Polyphenols extracted from puerarin (Pueraria lobata), resveratrol, and quercetin can mediate cholesterol efflux and intestinal microbial changes, thereby regulating bile acid excretion and reducing fat production. ${ }^{60,61}$ Scutellaria baicalensis can increase the expression of the rate-limiting enzyme cytochrome P450 family 7 subfamily A member 1 (CYP7A1) in the bile acid biosynthesis pathway, and CYP7A1 can prevent the obesity induced by a high-fat diet (the exact mechanism is not clear). ${ }^{62}$ Clove, allspice, and aromatic ginger extracts can induce TGR5-mediated BAS weight loss effects. Berberine affects the composition of intestinal microorganisms, activates multienzyme branched-chain ketoacid dehydrogenase complex (BCKDC) to promote BCAAs metabolism in the liver and IWAT of mice fed a HFD, and reduces the number of valine, leucine, isoleucine, and BCAAs during fasting by inhibiting the phosphorylation of branchedchain keto acid dehydrogenase E1 $\alpha$ subunit (BCKDHA) and multienzyme branched-chain $\beta$-keto dehydrogenase kinase (BCKDK), leading to bodyweight loss. ${ }^{63}$ Luffa cylindrica (L.) inhibits bacterial BCAAs biosynthesis, reduces BCAAs, and ameliorates BCAAs dysfunction, thus reducing obesity. ${ }^{64}$

\section{Regulate Hormone Level}

Endocrine cells can play roles in environmental balance and neuroendocrine function by secreting circulating hormones such as leptin, insulin, GLP1, PYY, cholecystokinin (CCK) and the hunger hormone, which can affect human eating behavior and signal satiety, playing a role in weight loss. ${ }^{65}$ Adipocytes can secrete adiponectin, leptin, and resistin. The most fruitful results in traditional Chinese medicine research on the regulation of hormone-induced weight loss were obtained from studies on leptin and adiponectin. 


\section{Enhance Leptin Sensitivity}

Leptin and its receptor (LEPR) are closely related to the regulation of energy uptake and consumption by the central nervous system. ${ }^{66}$ Leptin secreted by WAT can act on leptin-sensitive neurons in hypothalamic nuclei and regulate the lipid decomposition of WAT, nonshivering thermogenesis (NST) of BAT, lipid decomposition of beige adipose tissues (BeAT) and NST in brown adipose tissue through the sympathetic nervous system (SNS) ${ }^{67}$ Increasing leptinLEPR signaling and/or that of other neural pathways that activate leptin regulation can ameliorate obesity. Celastrol can inhibit food intake by increasing leptin sensitivity until body weight returns to normal. ${ }^{68}$ Astragaloside IV (ASIV) may improve the central leptin sensitivity of obese mice by upregulating the expression of LEPR mRNA and phosphorylation of signal transducer and activator of transcription 3 (p-STAT3) in the hypothalamus. ${ }^{69}$ Ginsenoside Rb1 increases leptin-JAK2-STAT3 signaling and leptininduced expression of brain-derived neurotrophic factor (BDNF) in the prefrontal cortex of obese mice, promotes leptin-BDNF-axonal growth and synaptogenesis, and plays a role in weight loss by improving leptin sensitivity. ${ }^{70}$ It can reverse central leptin resistance. ${ }^{70,71}$ Curcumin can improve the sensitivity to leptin by improving the hypoxic state of cells. ${ }^{72}$ Chaihu Shugan powder and the Linggui Zhugan decoction decrease the level of leptin and ameliorate obesity. ${ }^{73,74}$

\section{Regulate the Level of Adiponectin}

Adiponectin can reduce lipid accumulation in adipose tissue by activating the adiponectin-adenylate protein kinase pathway ${ }^{75,76}$ or activate BAT thermogenesis to regulate energy consumption. ${ }^{77}$ Piperonal (pepper) inhibits de novo adipogenesis in mice by increasing the level of circulating adiponectin mRNA, increasing the level of AMPK phosphorylation and S6K1 phosphorylation, and reducing the expression of SREBP1c and adipogenesisrelated mRNAs. ${ }^{78}$ Green tea (GT), which is rich in polyphenols, promotes BAT heat production by increasing adiponectin levels and activates AMPKal to promote lipid decomposition through PKA-HSL. ${ }^{79}$ 6-Gingerol can relieve ectopic lipid accumulation in the skeletal muscle of aging rats by increasing the level of adiponectin and its receptor, activating AdipoR1, and downstream AMPK/ PGC1 $\alpha$ signaling. ${ }^{80}$ Huganqingzhi tablets (HQTs) can increase adiponectin levels, activate AMPK and PPAR $\alpha$ pathways to inhibit SREBP-1 in the liver and de novo fat production, promote $\beta$-oxidation of fatty acids, and decrease cellular lipid levels. ${ }^{81}$ Sheng Jiang San (SJP) increases the level of serum adiponectin and inhibits the activation of inflammatory bodies and lipid accumulation through the adiponectin-AMPK pathway. ${ }^{82}$ Chaihu Shugan powder also reduces lipid synthesis by increasing the level of adiponectin. ${ }^{73}$

\section{Regulate the Levels of Other Hormones}

Among gastrointestinal hormones, GLP-1 and PYY are peripheral hormones associated with anorexia that can increase satiety, while ghrelin can induce hunger. ${ }^{83}$ Capsaicin can promote intestinal epithelial cells to secrete PYY and GLP-1, which inhibit the expression of neuropeptide Y (NPY) and agouti-related protein (AgRP), and activate neurons expressing POMC and CART in the hypothalamic $\mathrm{ARC}$, thus reducing food intake. ${ }^{46}$ Geniposide can ameliorate obesity by activating GLP-1 to activate AMPK $\alpha$ and Sirt1 in vivo (and in vitro). ${ }^{84}$ Lowpolar 22 Siraitia grosvenorii glycoside (L-SGgly) plays a role by increasing the level of serum GLP-1 in rats. ${ }^{85}$ Rhein increases plasma activity and the level of GLP-1, regulating appetite. ${ }^{86} \mathrm{~A}$ dietary supplement consisting of lemon fruit combined with hibiscus polyphenol extract increases the level of GLP-1 and decreases the level of ghrelin. ${ }^{87}$ The triterpenoids in Salvia miltiorrhiza may lead to weight loss by inhibiting the activity of ghrelin. ${ }^{88,89}$ Also, some traditional Chinese medicines can increase the production of gastrointestinal hormones (PYY, GLP-1, CCK, and ghrelin) through SCFAs and play a role in weight loss. Inulin increases the concentration of intestinal hormones and the level of SCFAs by increasing the density of specific intestinal endocrine cells in the colon and regulates the secretion of PYY and GLP-1, respectively, by free fatty acid receptor 2 (FFAR2)-dependent and independent mechanisms. ${ }^{49}$ Berberine can induce the expression and secretion of GLP-1 by upregulating SCFAs30 and stimulating GPR43, which can lead to weight loss. ${ }^{14}$

\section{Discussion}

We mainly explain the mechanism of weight loss of TCM according to the above three points, but the mechanism targeting muscle, liver and pancreas is not clear. These tissues play an important role in the metabolism of the human body. For example, insulin, a hormone secreted by pancreatic cells, can increase fat production during hyperinsulinemia. Therefore, in the early stage of increased insulin secretion, properly reducing the level of insulin 
can improve insulin sensitivity and achieve the purpose of weight loss. ${ }^{90}$ In addition, some studies have suggested that resveratrol ${ }^{91}$ can improve mitochondrial dysfunction, increase muscle content, reduce fat and lose weight. But it is not clear whether these herbs first improve obesity and then improve the metabolism of these tissues, or whether they first improve the metabolism of these tissues and then improve obesity.

A large number of studies in vitro and animal experiments have shown that TCM has the potential of multitarget treatment for obesity (Table 1). And in clinical treatment, traditional Chinese medicine physicians use TCM to prove its role in the treatment of obesity. In addition, the theory of food and drug homology made TCM, or named nutraceutical, ${ }^{92}$ an unrelated food drug derived from food that is believed to have physiological benefits and to improve chronic disease, more acceptable. This type of nutraceutical has great potential to be applied to human weight loss.

However, in clinical use, a combination of multiple drugs is often selected according to patients' personal conditions. The synergistic relationship ${ }^{93}$ among these drugs is complex, and the sample drug may have multiple action targets. Therefore, under the uncertain circumstances of the interaction between these drugs, the specific mechanism of single drugs, and the individual differences in the human body, it is still a very difficult and significant task to study the optimal dose, dose-effect relationship, half-life, and the treatment of related adverse reactions that can be used in clinical practice.

As obesity and obesity-related metabolic diseases are often multi-gene variation, multi-target disturbance and multi-disease related, it is not realistic to treat obesity in a single way, while TCM's multi-target, synergistic effect between drugs, small toxic and side effects, and easy to obtain from nature will become its advantages in treating obesity.

\section{Acknowledgments}

This work is supported by Pudong New Area Science and Technology Development Fund (PKJ2018-Y18) and Pudong New Area Municipal Health CommissionSummit Clinical Traditional Medicine Grant (PDZY2018-0602).

\section{Disclosure}

The authors declare that they have no conflicts of interest regarding this work or the publication of this article.

\section{References}

1. WHO. Obesity and overweight. 2018 Available from: https://www.who. int/news-room/fact-sheets/detail/obesity-and-overweight.. Accessed February 16, 2018.

2. Zhang X, Zhang M, Zhao Z, et al. Geographic variation in prevalence of adult obesity in China: results From the 2013-2014 National Chronic Disease and Risk Factor Surveillance. Ann Intern Med. 2019.

3. Dong Y, Ma J, Song Y, et al. Secular trends in blood pressure and overweight and obesity in chinese boys and girls aged 7 to 17 years from 1995 to 2014. Hypertension (Dallas, Tex: 1979). 2018;72 (2):298-305. doi:10.1161/HYPERTENSIONAHA.118.11291

4. Fan J-G, Kim S-U, Wong VW-S. New trends on obesity and NAFLD in Asia. J Hepatol. 2017;67(4):862-873. doi:10.1016/j.jhep.2017. 06.003

5. Booth JN 3rd, Li J, Zhang L, Chen L, Muntner P, Egan B. Trends in prehypertension and hypertension risk factors in US adults: 1999-2012. Hypertension. 2017;70(2):275-284. doi:10.1161/ HYPERTENSIONAHA.116.09004

6. Bray GA, Heisel WE, Afshin A, et al. The science of obesity management: an endocrine society scientific statement. Endocr Rev. 2018;39(2):79-132.

7. Goodarzi MO. Genetics of obesity: what genetic association studies have taught us about the biology of obesity and its complications. Lancet Diabetes Endocrinol. 2018;6(3):223-236. doi:10.1016/S22138587(17)30200-0

8. Ahmadizar F, Vijverberg SJ, Arets HG, et al. Childhood obesity in relation to poor asthma control and exacerbation: a meta-analysis. Eur Respir J. 2016;48(4):1063-1073. doi:10.1183/13993003.007662016

9. Di Angelantonio E, Bhupathiraju SN, Wormser D, et al. Body-mass index and all-cause mortality: individual-participant-data meta-analysis of 239 prospective studies in four continents. The Lancet. 2016;388 (10046):776-786. doi:10.1016/S0140-6736(16)30175-1

10. Hong M, Jung J, Park HS, et al. Shaofu Zhuyu decoction ameliorates obesity-mediated hepatic steatosis and systemic inflammation by regulating metabolic pathways. PLoS One. 2017;12(6):e0178514. doi: 10.1371 journal.pone. 0178514

11. Liu MT, Huang YJ, Zhang TY, Tan LB, Lu XF, Qin J. Lingguizhugan decoction attenuates diet-induced obesity and hepatosteatosis via gut microbiota. World J Gastroenterol. 2019;25(27):3590-3606.

12. Donaldson GP, Ladinsky MS, Yu KB, et al. Gut microbiota utilize immunoglobulin A for mucosal colonization. Science (New York, NY). 2018;360(6390):795-800. doi:10.1126/science.aaq0926

13. Ma X, Xu L, Alberobello AT, et al. Celastrol protects against obesity and metabolic dysfunction through activation of a HSF1-PGC1alpha transcriptional axis. Cell Metab. 2015;22(4):695-708. doi:10.1016/j. cmet.2015.08.005

14. Sun Y, Jin C, Zhang X, Jia W, Le J, Ye J. Restoration of GLP-1 secretion by Berberine is associated with protection of colon enterocytes from mitochondrial overheating in diet-induced obese mice. Nutr Diabetes. 2018;8(1):53. doi:10.1038/s41387-018-0061-x

15. Ding L, Li J, Song B, et al. Curcumin rescues high fat diet-induced obesity and insulin sensitivity in mice through regulating SREBP pathway. Toxicol Appl Pharmacol. 2016;304:99-109.

16. Xiao S, Zhang Z, Chen M, et al. Xiexin Tang ameliorates dyslipidemia in high-fat diet-induced obese rats via elevating gut microbiota-derived short chain fatty acids production and adjusting energy metabolism. $J$ Ethnopharmacol. 2019;241:112032. doi:10.1016/j.jep.2019.112032

17. Gong G, Han G, He H, Dong TTX, Tsim KWK, Zheng Y. An ancient chinese herbal decoction containing angelicae sinensis radix, astragali radix, jujuba fructus, and zingiberis rhizoma recens stimulates the browning conversion of white adipocyte in cultured 3T3-L1 Cells. Evid Based Complement Alternat Med. 2019;2019:3648685. doi: $10.1155 / 2019 / 3648685$ 
18. Viollet B, Guigas B, Leclerc J, et al. AMP-activated protein kinase in the regulation of hepatic energy metabolism: from physiology to therapeutic perspectives. Acta Physiol (Oxf). 2009;196(1):81-98.

19. Cheng CY, Yang AJ, Ekambaranellore P, Huang KC, Lin WW. Antiobesity action of INDUS810, a natural compound from Trigonella foenum-graecum: AMPK-dependent lipolysis effect in adipocytes. Obes Res Clin Pract. 2018;12(6):562-569. doi:10.1016/j.orcp.2018.08.005

20. Jiang D, Wang D, Zhuang X, et al. Berberine increases adipose triglyceride lipase in 3T3-L1 adipocytes through the AMPK pathway. Lipids Health Dis. 2016;15(1):214. doi:10.1186/s12944016-0383-4

21. Liu HK, Hung TM, Huang HC, et al. Bai-Hu-Jia-Ren-Shen-Tang decoction reduces fatty liver by activating AMP-activated protein kinase in vitro and in vivo. Evid Based Complement Alternat Med. 2015;2015:651734. doi:10.1155/2015/651734

22. Shen J, Niijima A, Tanida M, Horii Y, Maeda K, Nagai K. Olfactory stimulation with scent of grapefruit oil affects autonomic nerves, lipolysis and appetite in rats. Neurosci Lett. 2005;380(3):289-294. doi:10.1016/j.neulet.2005.01.058

23. Ono Y, Hattori E, Fukaya Y, Imai S, Ohizumi Y. Anti-obesity effect of Nelumbo nucifera leaves extract in mice and rats. J Ethnopharmacol. 2006;106(2):238-244. doi:10.1016/j.jep.2005.12.036

24. Murase T, Nagasawa A, Suzuki J, Hase T, Tokimitsu I. Beneficial effects of tea catechins on diet-induced obesity: stimulation of lipid catabolism in the liver. Int $J$ Obes Relat Metab Disord. 2002;26 (11):1459-1464. doi:10.1038/sj.ijo.0802141

25. Zhu H-J, Wang L-J, Wang X-Q, et al. Hormone-sensitive lipase is involved in the action of hydroxysafflor yellow A (HYSA) inhibiting adipogenesis of 3T3-L1cells. Fitoterapia. 2014;93:182-188. doi:10.1016/j.fitote.2014.01.001

26. Han MH, Jeong JS, Jeong JW, et al. Ethanol extracts of Aster yomena (Kitam.) Honda inhibit adipogenesis through the activation of the AMPK signaling pathway in 3T3-L1 preadipocytes. Drug Discov Ther. 2017;11(5):281-287. doi:10.5582/ddt.2017.01046

27. Chang C-J, Lin C-S, Lu -C-C, et al. Ganoderma lucidum reduces obesity in mice by modulating the composition of the gut microbiota. Nat Commun. 2017;12(1):7489. doi:10.1038/ncomms8489

28. Izdebska M, Herbet M, Gawrońska-Grzywacz M, et al. Resveratrol limits lipogenesis and enhance mitochondrial activity in HepG2 cells. J Pharm Pharmaceut Sci. 2018;21(1):504-515. doi:10.18433/jpps29994

29. Tran NKS, Kim GT, Park SH, Lee D, Shim SM, Park TS. Fermented cordyceps militaris extract prevents hepatosteatosis and adipocyte hypertrophy in high fat diet-fed mice. \#N/A. 2019;11:5.

30. Lone J, Choi JH, Kim SW, Yun JW. Curcumin induces brown fat-like phenotype in 3T3-L1 and primary white adipocytes. \#N/A. 2016;27:193-202.

31. Lim H, Park J, Kim HL, et al. Chrysophanic acid suppresses adipogenesis and induces thermogenesis by activating AMP-activated protein kinase alpha in vivo and in vitro. Front Pharmacol. 2016;7:476. doi:10.3389/fphar.2016.00476

32. Sheng D, Zhao S, Gao L, et al. BabaoDan attenuates high-fat diet-induced non-alcoholic fatty liver disease via activation of AMPK signaling. Cell Biosci. 2019;9:77. doi:10.1186/s13578-0190339-2

33. Baskaran P, Krishnan V, Ren J, Thyagarajan B. Capsaicin induces browning of white adipose tissue and counters obesity by activating TRPV1 channel-dependent mechanisms. Br J Pharmacol. 2016;173 (15):2369-2389. doi:10.1111/bph.13514

34. Bort A, Sanchez BG, Mateos-Gomez PA, Diaz-Laviada I, RodriguezHenche N. Capsaicin targets lipogenesis in HepG2 cells through AMPK activation, AKT inhibition and PPARs regulation. Int $J \mathrm{Mol}$ Sci. 2019;20:7. doi:10.3390/ijms20071660

35. Zu YX, Lu HY, Liu WW, et al. Jiang Gui Fang activated interscapular brown adipose tissue and induced epididymal white adipose tissue browning through the PPARgamma/SIRT1-PGC1alpha pathway. J Ethnopharmacol. 2020;248:112271. doi:10.1016/j.jep.2019.112271
36. Zhang C, Liu J, He X, et al. Caulis Spatholobi Ameliorates Obesity through Activating Brown Adipose Tissue and Modulating the Composition of Gut Microbiota. Int J Mol Sci. 2019;20:20.

37. Lee DH, Ahn J, Jang YJ, et al. Withania somnifera extract enhances energy expenditure via improving mitochondrial function in adipose tissue and skeletal muscle. \#N/A. 2020;12:2.

38. Kimura I, Ozawa K, Inoue D, et al. The gut microbiota suppresses insulin-mediated fat accumulation via the short-chain fatty acid receptor GPR43. Nat Commun. 2013;4:1829. doi:10.1038/ncomms2852

39. Scheppach W, Bartram P, Richter A, et al. Effect of short-chain fatty acids on the human colonic mucosa in vitro. JPEN Journal of Parenteral and Enteral Nutrition. 1992;16(1):43-48. doi:10.1177/ 014860719201600143

40. Yamashita H. Biological function of acetic acid-improvement in obesity and glucose tolerance by acetic acid in Type 2 diabetic rats. Crit Rev Food Sci Nutr. 2016;56(Suppl 1):S171-175. doi:10.1080/ 10408398.2015.1045966

41. Mollica MP, Mattace Raso G, Cavaliere G, et al. Butyrate regulates liver mitochondrial function, efficiency, and dynamics in insulin-resistant obese mice. Diabetes. 2017;66(5):1405-1418. doi:10.2337/db16-0924

42. Sanna S, van Zuydam NR, Mahajan A, et al. Causal relationships among the gut microbiome, short-chain fatty acids and metabolic diseases. Nat Genet. 2019;51(4):600-605. doi:10.1038/s41588-019-0350-x

43. Zhang B, Yue R, Chen Y, et al. Gut microbiota, a potential new target for chinese herbal medicines in treating diabetes mellitus. Evid Based Complement Alternat Med. 2019;2019:2634898.

44. Hu JL, Nie SP, Min FF, Xie MY. Polysaccharide from seeds of Plantago asiatica L. increases short-chain fatty acid production and fecal moisture along with lowering $\mathrm{pH}$ in mouse colon. J Agric Food Chem. 2012;60(46):11525-11532. doi:10.1021/jf302169u

45. Zhang X, Zhao Y, Zhang M, et al. Structural changes of gut microbiota during berberine-mediated prevention of obesity and insulin resistance in high-fat diet-fed rats. PLoS One. 2012;7(8):e42529. doi:10.1371/journal.pone.0042529

46. Wang Y, Tang C, Tang Y, Yin H, Liu X. Capsaicin has an anti-obesity effect through alterations in gut microbiota populations and short-chain fatty acid concentrations. Food Nutr Res. 2020;64.

47. Wang JH, Kim BS, Han K, Kim H. Ephedra-treated donor-derived gut microbiota transplantation ameliorates high fat diet-induced obesity in rats. Int J Env Res Public Health. 2017;14:6. doi:10.3390/ ijerph15010006

48. Zhang M, Zhao J, Deng J, Duan Z, Zhu C, Fan D. The protective effect of protopanaxatriol-type saponin on intestinal health in antibiotic-treated mice. Food Funct. 2019;10(7):4124-4133. doi:10.1039/C9FO00242A

49. Brooks L, Viardot A, Tsakmaki A, et al. Fermentable carbohydrate stimulates FFAR2-dependent colonic PYY cell expansion to increase satiety. Mol Metab. 2017;6(1):48-60. doi:10.1016/j. molmet.2016.10.011

50. Gao B, Wang R, Peng Y, Li X. Effects of a homogeneous polysaccharide from Sijunzi decoction on human intestinal microbes and short chain fatty acids in vitro. $J$ Ethnopharmacol. 2018;224:465-473. doi:10.1016/j.jep.2018.06.006

51. Wahlstrom A, Sayin SI, Marschall HU, Backhed F. Intestinal crosstalk between bile acids and microbiota and its impact on host metabolism. Cell Metab. 2016;24(1):41-50. doi:10.1016/j. cmet.2016.05.005

52. Tian Y, Gui W, Koo I, et al. The microbiome modulating activity of bile acids. Gut Microbes. 2020;1-18.

53. Chiang JY. Bile acid metabolism and signaling. Compr Physiol. 2013;3(3):1191-1212.

54. Velazquez-Villegas LA, Perino A, Lemos V, et al. TGR5 signalling promotes mitochondrial fission and beige remodelling of white adipose tissue. Nat Commun. 2018;9(1):245. doi:10.1038/s41467-01702068-0 
55. Ahmad TR, Haeusler RA. Bile acids in glucose metabolism and insulin signalling - mechanisms and research needs. Nat Rev Endocrinol. 2019;15(12):701-712.

56. Schenkel LC, Sivanesan S, Zhang J, et al. Choline supplementation restores substrate balance and alleviates complications of Pcyt2 deficiency. J Nutri Biochem. 2015;26(11):1221-1234. doi:10.1016/j. jnutbio.2015.05.014

57. Brown AL, Conrad K, Allende DS, et al. Dietary choline supplementation attenuates high-fat-diet-induced hepatocellular carcinoma in mice. J Nutr. 2020;150(4):775-783. doi:10.1093/jn/ nxz315

58. Cummings NE, Williams EM, Kasza I, et al. Restoration of metabolic health by decreased consumption of branched-chain amino acids. The Journal of Physiology. 2018;596(4):623-645.

59. Zhang F, Zhao S, Yan W, et al. Branched Chain amino acids cause liver injury in obese/diabetic mice by promoting adipocyte lipolysis and inhibiting hepatic autophagy. EBioMedicine. 2016;13:157-167. doi:10.1016/j.ebiom.2016.10.013

60. Huang F, Zheng X, Ma X, et al. Theabrownin from Pu-erh tea attenuates hypercholesterolemia via modulation of gut microbiota and bile acid metabolism. Nat Commun. 2019;10(1):4971.

61. Chambers KF, Day PE, Aboufarrag HT, Kroon PA. Polyphenol effects on cholesterol metabolism via bile acid biosynthesis, CYP7A1: a review. \#N/A. 2019;11:11.

62. Han $\mathrm{K}$, Bose $\mathrm{S}$, Wang $\mathrm{JH}$, et al. In vivo therapeutic effect of combination treatment with metformin and Scutellaria baicalensis on maintaining bile acid homeostasis. PLoS One. 2017;12(9):e0182467. doi:10.1371/journal.pone.0182467

63. Yue SJ, Liu J, Wang AT, et al. Berberine alleviates insulin resistance by reducing peripheral branched-chain amino acids. Am $J$ Physiol Endocrinol Metab. 2019;316(1):E73-E85. doi:10.1152/ ajpendo.00256.2018

64. Zhang L, Yue Y, Shi M, et al. Dietary Luffa cylindrica (L.) Roem promotes branched-chain amino acid catabolism in the circulation system via gut microbiota in diet-induced obese mice. Food Chem. 2020;320:126648. doi:10.1016/j.foodchem.2020.126648

65. Steinert RE, Feinle-Bisset C, Asarian L, Horowitz M, Beglinger C, Geary N. Ghrelin, CCK, GLP-1, and PYY(3-36): secretory controls and physiological roles in eating and glycemia in health, obesity, and after RYGB. Physiol Rev. 2017;97(1):411-463. doi:10.1152/ physrev.00031.2014

66. Pan WW, Myers MG Jr. Leptin and the maintenance of elevated body weight. \#N/A. 2018;19(2):95-105.

67. Caron A, Lee S, Elmquist JK, Gautron L. Leptin and brain-adipose crosstalks. \#N/A. 2018;19(3):153-165.

68. Liu J, Lee J, Salazar Hernandez MA, Mazitschek R, Ozcan U. Treatment of obesity with celastrol. Cell. 2015;161(5):999-1011. doi:10.1016/j.cell.2015.05.011

69. Jiang $\mathrm{P}$, Ma D, Wang $\mathrm{X}$, et al. Astragaloside IV prevents obesity-associated hypertension by improving pro-inflammatory reaction and leptin resistance. Mol Cells. 2018;41(3):244-255.

70. Wu Y, Huang X-F, Bell C, Yu YY. Ginsenoside Rb1 improves leptin sensitivity in the prefrontal cortex in obese mice. CNS Neurosci Ther. 2018;24(2):98-107. doi:10.1111/cns.12776

71. Zhou P, Xie W, He S, et al. Ginsenoside Rb1 as an anti-diabetic agent and its underlying mechanism analysis. Cells. 2019;8:3. doi:10.3390/ cells8030204

72. Chang C-J, Lu -C-C, Lin C-S, et al. Antrodia cinnamomea reduces obesity and modulates the gut microbiota in high-fat diet-fed mice. Int $J$ Obes (Lond). 2018;42(2):231-243. doi:10.1038/ijo.2017.149

73. Jiang WN, Li D, Jiang T, et al. Protective effects of chaihu shugan san () on nonalcoholic fatty liver disease in rats with insulin resistance. Chin J Integr Med. 2018;24(2):125-132. doi:10.1007/ s11655-016-2252-4
74. Yao L, Wei J, Shi S, et al. Modified lingguizhugan decoction incorporated with dietary restriction and exercise ameliorates hyperglycemia, hyperlipidemia and hypertension in a rat model of the metabolic syndrome. BMC Complement Altern Med. 2017;17(1):132. doi:10.1186/s12906-017-1557-y

75. Akingbemi BT. Adiponectin receptors in energy homeostasis and obesity pathogenesis. Prog Mol Biol Transl Sci. 2013;114:317-342.

76. Iwabu M, Yamauchi T, Okada-Iwabu M, et al. Adiponectin and AdipoR1 regulate PGC-1alpha and mitochondria by $\mathrm{Ca}(2+)$ and AMPK/SIRT1. Nature. 2010;464(7293):1313-1319. doi:10.1038/ nature08991

77. Sun L, Yan J, Goh HJ, et al. Fibroblast Growth Factor-21, leptin, and adiponectin responses to acute cold-induced brown adipose tissue activation. J Clin Endocrinol Metab. 2020;105:3. doi:10.1210/ clinem/dgaa005

78. Li X, Choi Y, Yanakawa Y, Park T. Piperonal prevents high-fat diet-induced hepatic steatosis and insulin resistance in mice via activation of adiponectin/AMPK pathway. Int $J$ Obes (Lond). 2014;38(1):140-147. doi:10.1038/ijo.2013.70

79. Bolin AP, Sousa-Filho CPB, Marinovic MP, Rodrigues AC, Otton R. Polyphenol-rich green tea extract induces thermogenesis in mice by a mechanism dependent on adiponectin signaling. \#N/A. 2020;78:108322.

80. Liu L, Yao L, Wang S, et al. 6-Gingerol improves ectopic lipid accumulation, mitochondrial dysfunction, and insulin resistance in skeletal muscle of ageing rats: dual stimulation of the AMPK/ PGC-1alpha signaling pathway via plasma adiponectin and muscular AdipoR1. Mol Nutr Food Res. 2019;63(6):e1800649. doi:10.1002/ mnfr.201800649

81. Yin J, Luo Y, Deng H, et al. Hugan Qingzhi medication ameliorates hepatic steatosis by activating AMPK and PPARalpha pathways in L02 cells and HepG2 cells. J Ethnopharmacol. 2014;154(1):229-239. doi:10.1016/j.jep.2014.04.011

82. Miao YF, Li J, Zhang YM, et al. Sheng-jiang powder ameliorates obesity-induced pancreatic inflammatory injury via stimulating activation of the AMPK signalling pathway in rats. World $J$ Gastroenterol. 2018;24(39):4448-4461. doi:10.3748/wjg.v24. i39.4448

83. Schellekens H, Finger BC, Dinan TG, Cryan JF. Ghrelin signalling and obesity: at the interface of stress, mood and food reward. Pharmacol Ther. 2012;135(3):316-326. doi:10.1016/j. pharmthera.2012.06.004

84. Ma Z-G, Kong C-Y, Song P, Zhang X, Yuan Y-P, Tang Q-Z. Geniposide protects against obesity-related cardiac injury through AMPK $\alpha$ - and Sirt1-dependent mechanisms. Oxid Med Cell Longev. 2018;2018:1-12. doi:10.1155/2018/8938207

85. Zhang Y, Zhou G, Peng Y, Wang M, Li X. Anti-hyperglycemic and anti-hyperlipidemic effects of a special fraction of Luohanguo extract on obese T2DM rats. $J$ Ethnopharmacol. 2020;247:112273. doi:10.1016/j.jep.2019.112273

86. Wang R, Zang P, Chen J, et al. Gut microbiota play an essential role in the antidiabetic effects of rhein. Evid Based Complement Alternat Med. 2018;2018:6093282. doi:10.1155/2018/6093282

87. Boix-Castejon M, Herranz-Lopez M, Perez Gago A, et al. Hibiscus and lemon verbena polyphenols modulate appetite-related biomarkers in overweight subjects: a randomized controlled trial. Food Funct. 2018;9(6):3173-3184. doi:10.1039/C8FO00367J

88. Tung NH, Nakajima K, Uto T, et al. Bioactive Triterpenes from the Root of Salvia miltiorrhiza Bunge. Phytother Res. 2017;31 (9):1457-1460. doi:10.1002/ptr.5877

89. Nakajima K, Oiso S, Uto T, Morinaga O, Shoyama Y, Kariyazono H. Triterpenes suppress octanoylated ghrelin production in ghrelin-expressing human gastric carcinoma cells. Biomedical Research (Tokyo, Japan). 2016;37(6):343-349. doi:10.2220/ biomedres.37.343 
90. Page MM, Johnson JD. Mild suppression of hyperinsulinemia to treat obesity and insulin resistance. Trends Endocrinol Metab. 2018;29 (6):389-399. doi:10.1016/j.tem.2018.03.018

91. Huang Y, Zhu X, Chen K, et al. Resveratrol prevents sarcopenic obesity by reversing mitochondrial dysfunction and oxidative stress via the PKA/LKB1/AMPK pathway. Aging (Albany NY). 2019;11 (8):2217-2240. doi:10.18632/aging.101910
92. Scicchitano P, Cameli M, Maiello M, et al. Nutraceuticals and dyslipidaemia: beyond the common therapeutics. J Funct Foods. 2014;6:11-32. doi:10.1016/j.jff.2013.12.006

93. Shoba G, Joy D, Joseph T, Majeed M, Rajendran R, Srinivas PS. Influence of piperine on the pharmacokinetics of curcumin in animals and human volunteers. Planta Med. 1998;64(4):353-356. doi:10.1055/s-2006-957450

Diabetes, Metabolic Syndrome and Obesity: Targets and Therapy

\section{Publish your work in this journal}

Diabetes, Metabolic Syndrome and Obesity: Targets and Therapy is an international, peer-reviewed open-access journal committed to the rapid publication of the latest laboratory and clinical findings in the fields of diabetes, metabolic syndrome and obesity research. Original research, review, case reports, hypothesis formation, expert opinion and commentaries are all considered for publication. The manuscript management system is completely online and includes a very quick and fair peer-review system, which is all easy to use. Visit http://www.dovepress.com/testimonials.php to read real quotes from published authors. 